\begin{tabular}{|c|c|c|c|}
\hline \multicolumn{4}{|c|}{ Abstract 16 Table 1} \\
\hline & HF CUINIC ( $\mathrm{n}=161)$ & NO HF CUINIC ( $\mathrm{n}=406)$ & $p$ value \\
\hline Age & $78 \pm 9$ & $80 \pm 9$ & 0.02 \\
\hline Average NTproBNP & 3352 & 2365 & $<0.0001$ \\
\hline $\begin{array}{l}\text { All-cause } \\
\text { hospitalisation }\end{array}$ & $37(22.3 \%)$ & $180(44.3 \%)$ & $<0.0001$ \\
\hline CV hospitalisation & $4(2.5 \%)$ & $57 \quad(14.0 \%)$ & $<0.0001$ \\
\hline Deaths & $9(5.6 \%)$ & 33 (8.1\%) & 0.3 \\
\hline Mean hospital stay & 4.7 days & 5.6 days & 0.42 \\
\hline
\end{tabular}

of this study was to compare hospitalisation and mortality rates in patients with suspected HF and raised NTproBNP who were reviewed in a HF clinic to those who were not referred.

Methods Data were collected retrospectively from hospital records. All patients with a raised NTproBNP (>400 pg/ml) from February to September 2014 were identified via the Trust biochemistry laboratory and were included in the study. These patients were divided into those referred to and reviewed in the clinic (HF Clinic) and those who were not referred (No HF Clinic). Hospital coding data, information services and case notes were used to identify hospital admissions, length of stay, reason for admission and mortality in all patients over a minimum follow up period of 6-months from the time of the raised NTproBNP result. Comparisons were then made between the HF clinic and No HF clinic cohorts.

Results 567 consecutive patients were identified: 161 (28\%) in the HF Clinic group and $406(72 \%)$ in the No HF Clinic group. All HF clinic patients were seen within 2 weeks (NTproBNP > $2000 \mathrm{pg} / \mathrm{ml}$ ) or 6 weeks (NTproBNP 400$2000 \mathrm{pg} / \mathrm{ml}$ ) in line with NICE recommendations. Mean follow up period was $8.9 \pm 2.3$ months. Mean patient age was $80 \pm 9$ years. The HF clinic cohort was slightly younger (Table 1). 52\% of HF clinic patients were male versus $48 \%$ of No HF clinic patients $(p=0.42$ ). Despite having a significantly higher NTproBNP level HF clinic patients had a much lower rate of all cause and cardiovascular admissions than the No HF clinic patients (Table 1 and Figure 1). There was no difference in mortality.

Conclusions Hospitalisation rates in patients with suspected heart failure and raised NTproBNP were extremely high over a relatively short follow up period. Patients reviewed in a specialist HF clinic had much higher NTproBNP levels, suggesting they were at higher risk of adverse outcomes, yet also had significantly lower rates of all cause and cardiovascular hospitalisation. The data are observational so causation and association cannot be separated however the magnitude of the difference between the patient groups suggests referral to HF clinics may improve outcomes. Our findings support implementation of the relevant NICE guidelines for patients with suspected $\mathrm{HF}$ and higher rates of referral from primary care should be encouraged.

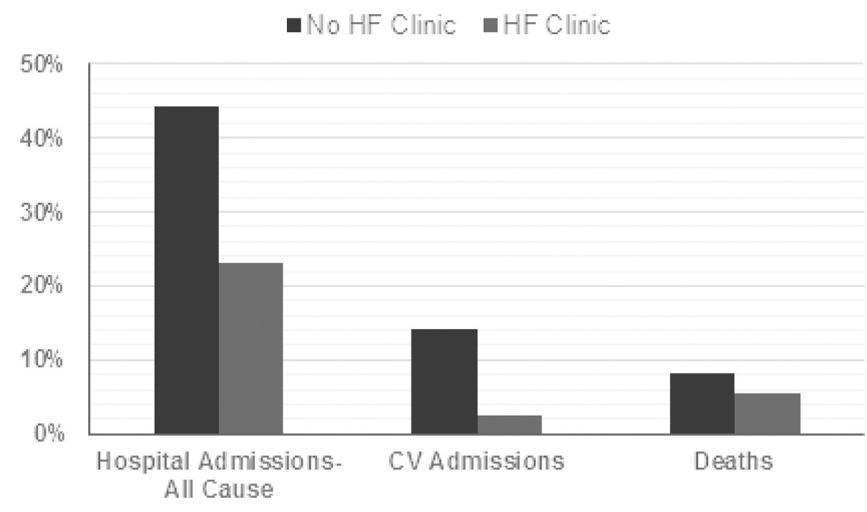

Abstract 16 Figure 1

\section{TELEMEDICINE IN PATIENTS WITH NEW DIAGNOSIS OF HEART FAILURE: FROM CLINICAL RESEARCH TO PRACTICE}

${ }^{1}$ George Koulaouzidis*, ${ }^{2}$ Kevin Mohee, ${ }^{3}$ David Barrett, ${ }^{2}$ Andrew Clark. ${ }^{1}$ Royal Brompton Hospital; ${ }^{2}$ Department of Academic Cardiology, Castle Hill Hospital; ${ }^{3}$ Faculty of Health and Social Care, University of Hull; *Presenting Author

\subsection{6/heartjnl-2016-309890.17}

Background Heart failure (HF) is a complex clinical syndrome associated with high mortality and high rate of hospital readmissions. Telehealth $(\mathrm{TH})$ is a promising strategy for improving HF outcomes but there is an urgent need to properly identify those patients in whom a TH approach would provide benefit. The purpose of this study was to determine if $\mathrm{TH}$ in patients with recently diagnosed HF and ejection fraction $<45 \%$, reduces the risk of re-admission from any cause or death from any cause.

Methods A retrospective study of 124 patients (78.2\% male; $68.6 \pm 12.6$ years; $56.5 \%$ ischaemic aetiology) who underwent $\mathrm{TH}$ and 345 patients $(68.5 \%$ male; $70.2 \pm 10.7$ years; $56.3 \%$ ischaemic aetiology) who underwent the usual-care (UC), between 2009-12. All patients had a recent diagnosis of HF, NHYA class II-III and ejection fraction $<45 \%$. The TH group were assessed by body weight, blood pressure and heart rate on a daily basis using electronic devices with automatic transfer of measured data to an online database. The followup period was 12 months.

Results Death from any cause occurred in $8.1 \%$ of the $\mathrm{TH}$ group and $19 \%$ of the UC group, $p=0.002$. Readmissions for any cause occurred in $63.7 \%$ of patients in the $\mathrm{TH}$ group and $62.5 \%$ of patients in the UC group, $p=0.8$. The number of readmissions/patients was also similar in the two groups, $1.3 \pm 1.7$ in the TH group and $1.4 \pm 1.7$ in the UC group $(p=0.9)$. A difference in the number of days in hospital was noted $(8.1 \pm 12.8$ days in $\mathrm{TH}$ group and $9.5 \pm 17.3$ in UC group) but this was not statistically significant $(p=$ $0.4)$. There was significant difference in the days alive and away from the hospital in the two groups: $348.3 \pm 5.5$ days in the TH group and $329.9 \pm 4.6$ days in the UC group ( $p$ $=0.008)$.

Conclusions In patients with a recent diagnosis of $\mathrm{HF}$ and reduced left ventricle systolic function, $\mathrm{TH}$ is associated with lower any-cause mortality. Furthermore TH has the potential to reduce number of days lost to hospitalisation and death. 